\title{
Correction: FOXO3a represses VEGF expression through FOXM1- dependent and -independent mechanisms in breast cancer
}

\author{
C. T. Karadedou ${ }^{1}$ - A. R. Gomes ${ }^{1}$ - J. Chen ${ }^{1,2} \cdot$ M. Petkovic ${ }^{1} \cdot$ K.-K. Ho ${ }^{1} \cdot$ A. K. Zwolinska ${ }^{1}$ - A. Feltes ${ }^{1} \cdot$ S. Y. Wong ${ }^{2}$ \\ K. Y. K. Chan ${ }^{2}$ - Y.-N. Cheung ${ }^{2}$ • J. W. H. Tsang ${ }^{3}$ • J. J. Brosens ${ }^{1}$ • U.-S. Khoo ${ }^{2}$ E. W.-F. Lam ${ }^{1}$
}

Published online: 13 March 2019

๔ Springer Nature Limited 2019

\section{Correction to: Oncogene \\ https://doi.org/10.1038/onc.2011.368, \\ Published online 22 Aug 2011}

In the published version of this article, the images for cytoplasmic and nuclear FGF7 in MDA-MB-231 cells were duplicated and mistaken for total FGF7 in SKBR-3 and MDA-MB-231 cells.
The FGF7 was used here as an extra positive control for VEGF and not absolutely needed. Moreover, the total FGF7 was in fact redundant as the cytoplasmic and nuclear FGF7 was already shown in Fig. 2a. We do not believe that this mistake would have any consequence on the original review process, or the scientific conclusions reached in the article.

This correction notice contains the original blot, annotated, and shown in its totality, and the complete corrected Fig 2a.

These authors contributed equally: C. T. Karadedou, A. R. Gomes

These authors jointly supervised this work: C. T. Karadedou, A. R. Gomes

The original article can be found online at https://doi.org/10.1038/onc. 2011.368

$\triangle$ E. W.-F. Lam

eric.lam@imperial.ac.uk

1 Department of Surgery and Cancer, Cancer Research-UK Labs, Imperial College London, Hammersmith Hospital Campus, London, UK

2 Department of Pathology, The University of Hong Kong, Hong Kong SAR, China

3 Department of Clinical Oncology Li Ka Shing Faculty of Medicine, The University of Hong Kong, Hong Kong SAR, China 


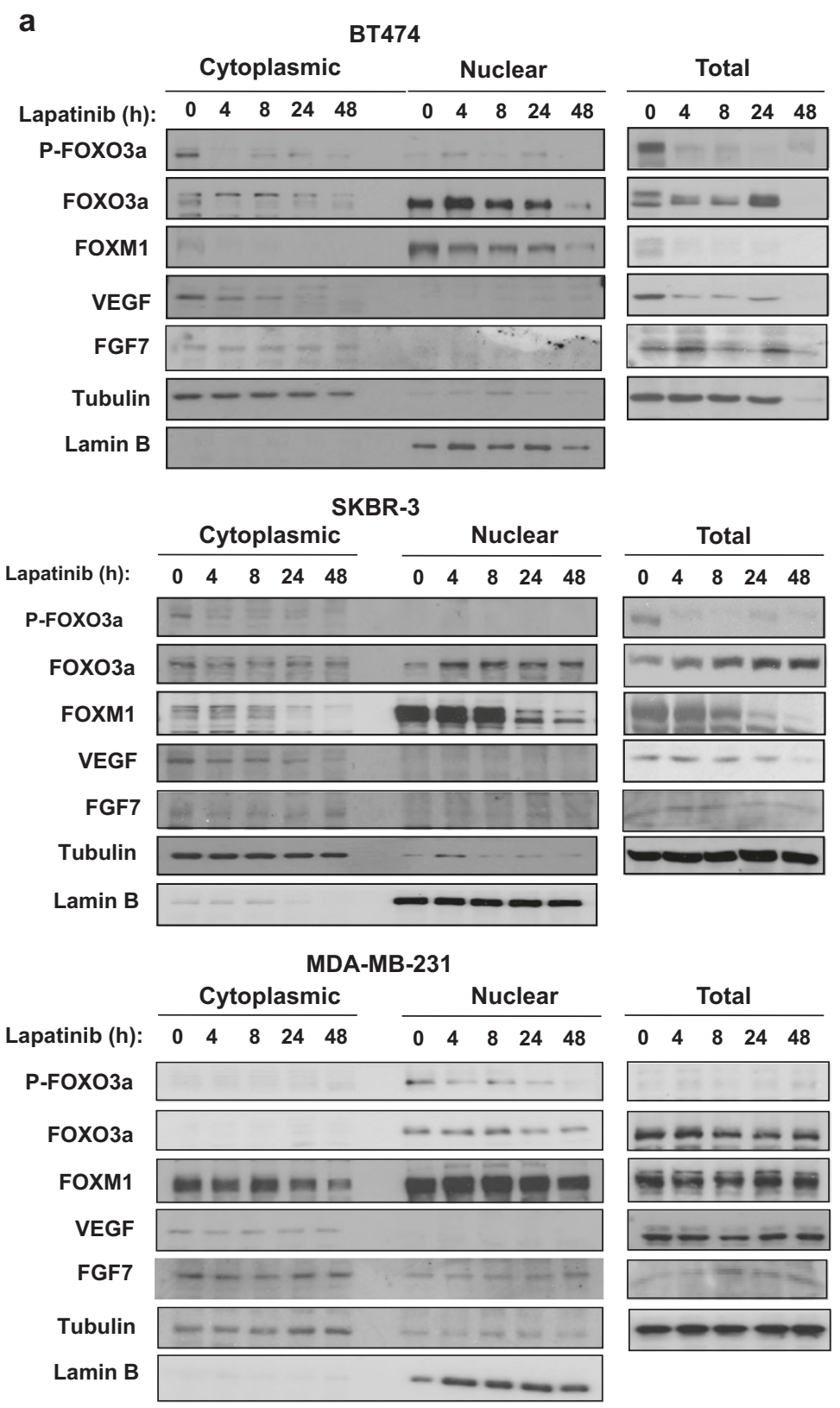

Fig. 2 Expression of FOXO3a, FOXM1 and VEGF in response to lapatinib treatment in breast cancer cell lines. The lapatinib-sensitive BT474 and SKBR-3 and resistant MDA-MB-231 cells were cultured in $10 \%$ foetal calf serum medium for 24 ?h before treatment with lapatinib. (a) At times indicated, cells were collected and analysed for PFOXO3a, total FOXO3a, FOXM1, VEGF, FGF7, LaminB and tubulin expression by western blotting of the nuclear/cytoplamic (left panel) and total (right panel) lysates. (b) VEGF concentrations in supernatants of the lapatinib-treated breast cancer cells were measured by a quantitative sandwich enzyme immunoassay according to the manufacturer's protocol (Quantikine enzyme-linked immunosorbent assay, R\&D Systems, Abingdon, UK). The optical density was measured at 450?nm using a Sunrise-Tecan plate reader (TECAN Ltd., Reading, UK) and VEGF concentrations normalised using standard curves. (c) In parallel, VEGF mRNA levels of these lapatinib-treated breast cancer cells were also analysed by quantitative real-time (qRT)-PCR and normalised to L19 RNA expression. Total RNA (2? $\mu \mathrm{g}$ ) isolated using the RNeasy
Mini kit (Qiagen) was reverse transcribed using the Superscript III reverse transcriptase and random primers (Invitrogen, Paisley, UK), and the resulting first strand?cDNA was used as template in the RT-PCR. All experiments were performed in triplicate. The following genespecific primer pairs were designed using the ABI Primer Express software: FOXM1-sense: 5'-TGCAGCTAGGGATGTGAATCTTC-3' and FOXM1-antisense: 5'-GGAGCCCAGTCCATCAGAACT-3'; ERa-sense: 5'-CAGATGGTCAGTGCCTTGTTGG-3' and ERa-antisense: 5'-CCAAGAGCAAGTTAGGAGCAAACAG-3'; L19-sense 5'GCGGAAGGGTACAGCCAAT-3' and L19-antisense 5'-GCAGCCG GCGCAAA-3'. Specificity of each primer was determined using NCBI BLAST module. RT-PCR was performed with ABI PRISM 7700 Sequence Detection System using SYBR Green Mastermix (Applied Biosystems, Brackley, UK). The RT-qPCR results shown are representative of three independent experiments. FOXM1 mRNA levels of these cells were also analysed by RT-qPCR, and normalised with L19 RNA expression 